\title{
Engineering Fano resonances in discrete arrays
}

\author{
Andrey E. Miroshnichenko and Yuri S. Kivshar \\ Nonlinear Physics Centre and Centre for Ultra-high bandwidth Devices for Optical Systems (CUDOS), Research School \\ of Physical Sciences and Engineering, Australian National University, Canberra ACT 0200, Australia
}

(Received 2 May 2005; revised manuscript received 28 June 2005; published 23 November 2005)

\begin{abstract}
We study transmission properties of discrete arrays composed of a linear waveguide coupled to a system of $N$ side defect states. This simple system can be used to model discrete networks of coupled defect modes in photonic crystals, complex waveguide arrays in two-dimensional nonlinear lattices, and ring-resonator structures. We demonstrate the basic principles of the resonant scattering management through engineering Fano resonances and find exact results for the wave transmission coefficient. We reveal conditions for perfect reflections and transmissions due to either destructive or constructive interferences, and associate them with Fano resonances, also demonstrating how these resonances can be tuned by nonlinear defects.
\end{abstract}

DOI: 10.1103/PhysRevE.72.056611

\section{INTRODUCTION}

During the past decade we observe a growing interest in theoretical and experimental studies of different types of resonant wave phenomena associated with either direct or indirect manifestation of the classical Fano resonance [1] in nanoscale devices with side-coupled waveguiding structures [2-10]. These structures can be presented as one or more waveguides in which forward and backward propagating waves are indirectly coupled to each other via one or more mediating resonant cavities or defect states. The well-known systems for realizing these structures are based on a straight photonic-crystal waveguide with a number of side defect modes [11], microring resonator structures in which two channel waveguides are side-coupled to microring resonators [12] or variety of bend photonic-crystal waveguides [13]. Similar structures can be created in the discrete networks extensively discussed for routing and switching of discrete optical solitons [14].

In all such structures, the forward and backward propagating modes within the waveguide are coupled via the defects; the transmission becomes highly sensitive to the resonant properties of the defect states, and it is usually associated with the so-called Fano resonances. Indeed, the underlying physics of the Fano resonances finds its origin in wave interference which occurs in the systems characterized by one or several discrete energy states that interact with the continuum spectrum. In the corresponding transmission dependencies, the interference effect leads to either perfect transmission or perfect reflection, producing a sharp asymmetric response. This kind of the wave resonance is also common in different interferometer devices such as the Aharonov-Bohm interferometer [15] and the Mach-Zehnder interferometer [16].

One of the simplest models that can be used to study the Fano resonances in discrete networks as well as the resonant coupling and interaction between discrete degrees of freedom and a continuum spectrum is the so-called FanoAnderson model [17]. It describes a linear array of coupled elements (e.g., effective particles or defect modes) with the nearest-neighbor interaction coupled to one or several defect states through a local coupling. Such a simple discrete model allows to describe the basic physics of the Fano resonances in a rather simple way including the nonlinear and bistability regimes [18]. In particular, this model allows to derive analytical results for the wave transmission and reflection, and it may serve as a guideline for the analysis of more complicated physical models associated with the Fano resonance.

In this paper, we study the transmission properties of discrete networks composed of linear arrays of interacting elements coupled to systems of $N$ side defects described by the generalized discrete Fano-Anderson model. This model allows us to find exact solutions for the wave transmission coefficient and the conditions for the perfect reflections and transmissions due to either destructive or constructive interference. Using these results, we demonstrate and explain the basic principles of the resonant scattering under the condition of the Fano resonances, and also suggest the concept of the Fano resonance engineering. In particular, for several different examples we demonstrate that in the presence of a defect the destructive wave interference remains always resonant, while the constructive wave interference could be or could not be resonant. As a result, this brings us to the general conclusion that the main feature of the Fano resonance is the resonant reflection but not transmission. We also demonstrate how the Fano resonances can be tuned by introducing nonlinear defects into a discrete network. The results are quite general and can be applied to different physical systems such as quantum dots or photonic crystal waveguides, for example.

The paper is organized as follows. In Sec. II we introduce our discrete model describing a linear chain with $N$ defects and describe the main features of the linear wave transmission. In particular, we define the conditions for both resonant transmission and reflection due to the interaction with a side chain of $N$ defects coupled locally to the main array. In Sec. III we demonstrate how the Fano resonance in a linear system can be tuned by introducing nonlinear defects. Finally, Sec. IV concludes the paper.

\section{LINEAR TRANSMISSION}

We consider the generalized linear Fano-Anderson model that describes an infinite array of interacting elements (e.g., 


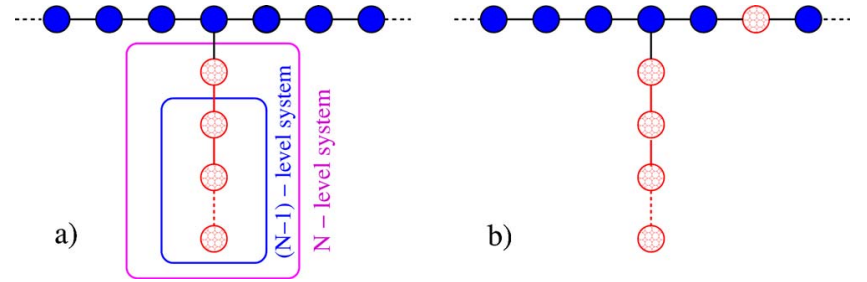

FIG. 1. (Color online) Schematic view of the generalized FanoAnderson model with a locally coupled $N$-defect chain without (a) or with (b) additional defects in the main array.

effective particles) coupled locally to a complex side defect (the so-called Fano defect) characterized by more than one degrees of freedom, as shown schematically in a specific example of Fig. 1(a). From the viewpoint of the Fano resonance, each degree of freedom of the defect chain contributes with an additional local path for the wave scattering, or, in other words, generates an additional discrete state. Each discrete state leads to the possibility of additional interference condition, so that the presence of several defects may show a variety of interference phenomena. In order to study these effects in details, both analytically and numerically, we take one of the simplest implementations of the $N$-mode Fano defect as a finite chain of defects with the nearest-neighbor coupling between them; see Fig. 1(a) [19].

We then provide with specific example from the theory of photonic crystal (PC) waveguides in order to show how this model can be implemented to real physical system.

\section{A. Model for $N$-defect Fano resonances}

We start our study from the analysis of the linear transmission when the Hamiltonian of the model can be written in the following form

$$
H_{L}=H_{0}+H_{F}+H_{0 F},
$$

where

$$
\begin{gathered}
H_{0}=C \sum_{n}\left(\phi_{n} \phi_{n+1}^{*}+\text { c.c. }\right) \\
H_{F}=\sum_{m=1}^{N-1}\left(E_{m}\left|\varphi_{m}\right|^{2}+V_{m} \varphi_{m} \varphi_{m+1}^{*}+\text { c.c. }\right)+E_{N}\left|\varphi_{N}\right|^{2}, \\
H_{0 F}=V_{0} \phi_{0} \varphi_{1}^{*}+\text { c.c. },
\end{gathered}
$$

and the asterisk stands for the complex conjugation. The model with the Hamiltonian (1), (2) describes the interaction of two subsystems coupled locally to each other at a single site [see Fig. 1(a)]. One subsystem is an infinite homogeneous array of equivalent elements described by the wave functions $\phi_{n}$ with the strength of the nearest-neighbor interaction characterized by the parameter $C$. In this array, waves propagate freely and they are characterized by the dispersion relation $\omega_{q}=2 C \cos q$. The other subsystem is a finite inhomogeneous chain of $N$ elements described by the wave functions $\varphi_{n}$ which acts as a complex localized defect attached to the main array, with $E_{m}$ being the energy associated with the $m$ th element. We assume that the defect sites are coupled through the nearest-neighbor interaction with the strength $V_{m}$.

Depending on the ratio of the coupling coefficients $C$ and $V_{0}$ this model can be directly applied to different physical systems. For example, in quantum $\operatorname{dots} C \ll V_{0}$ [2-4], in photonic-crystal waveguides coupling coefficients $C$ and $V_{0}$ are of the same order $[5-7,13]$, for scattering by timeperiodic and spatially localized states (discrete breathers) even the case $C<V_{0}$ might be possible [20]. Therefore, we will not concentrate on a particular type of the physical system and will present generic results.

From the Hamiltonian (1) and (2) we can derive the equations of motion in the frequency domain,

$$
\begin{gathered}
\omega \phi_{n}=C\left(\phi_{n-1}+\phi_{n+1}\right)+V_{0} \varphi_{1} \delta_{n, 0}, \\
\omega \varphi_{1}=E_{1} \varphi_{1}+V_{0} \phi_{0}+V_{1} \varphi_{2}, \\
\omega \varphi_{2}=E_{2} \varphi_{2}+V_{1} \varphi_{1}+V_{2} \varphi_{3}, \\
\vdots \\
\omega \varphi_{N}=E_{N} \varphi_{N}+V_{N-1} \varphi_{N-1},
\end{gathered}
$$

and obtain a simple recurrence relation for $\varphi_{m}$,

$$
\varphi_{N-l}=\frac{V_{N-l-1} \rho_{l}(\omega)}{\rho_{l+1}(\omega)} \varphi_{N-l-1},
$$

where

$$
\rho_{l}(\omega)=\operatorname{det}\left[\omega I-H_{F}^{l}\right]
$$

is a characteristic polynomial of the subsystem of $l$ sites only (calculated from the site $N-l+1$ to the site $N$ )

$$
H_{F}^{l}=\left(\begin{array}{ccccc}
E_{N} & V_{N-1} & 0 & \cdots & 0 \\
V_{N-1} & E_{N-1} & V_{N-2} & \cdots & 0 \\
\vdots & & \ddots & & \vdots \\
0 & \cdots & 0 & V_{N-l+1} & E_{N-l+1}
\end{array}\right)
$$

and $I$ is the identity matrix $l \times l$.

The recurrence relation (4) is valid for $l=0, \ldots, N-2$ and, for simplicity, we assume that $\rho_{0}(\omega) \equiv 1$. By writing the relation (4) for $\varphi_{2}$,

$$
\varphi_{2}=\frac{V_{1} \rho_{N-2}(\omega)}{\rho_{N-1}(\omega)} \varphi_{1}
$$

and substituting it into the second equation of (3), we extend the recurrence relation (4) to the following form

$$
\varphi_{1}=\frac{V_{0} \rho_{N-1}(\omega)}{\rho_{N}(\omega)} \phi_{0},
$$

which allows us to eliminate additional degrees of freedom from the system (3) and obtain a system of equations for describing the wave propagation in the main array only 

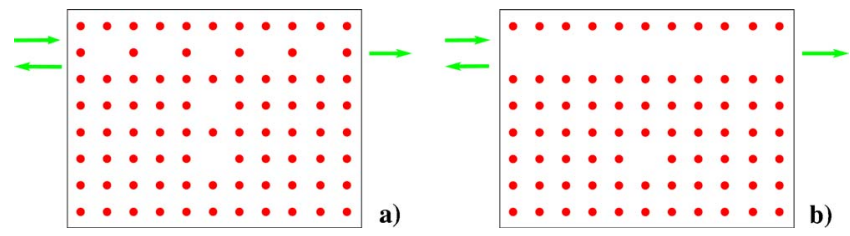

FIG. 2. (Color online) Schematic view of two photonic crystal waveguide configurations with side-coupled defects. The waveguides and defects are constructed by removing some rods in a periodic structure. These two examples show that coupling between defect rods in straight waveguide and coupling to side-coupled defects can be easily tuned.

$$
\omega \phi_{n}=C\left(\phi_{n-1}+\phi_{n+1}\right)+\frac{\rho_{N-1}(\omega)}{\rho_{N}(\omega)} V_{0}^{2} \phi_{0} \delta_{n, 0}
$$

with an effective localized defect. This defect acts as a scattering potential whose strength depends on the frequency of the incoming wave (8). The function $\rho_{l}(\omega)$ vanishes when the frequency $\omega$ coincides with one of the eigenfrequencies of the corresponding subsystem of $l$ sites described by Eq. (6). Therefore, our first important result is that, in general, there exist $N-1$ frequencies where the strength of the defect vanishes and the induced scattering potential becomes transparent, and $N$ frequencies where the strength of the defect will become infinite making the scattering potential opaque.

\section{B. Examples from the photonic crystal theory}

One of the important physical systems, where the model described above can be applied, is photonic crystal waveguides. Some examples are shown in Fig. 2.

Photonic crystals are artificial dielectric structures with a periodic modulation in the refractive index that create regions of forbidden frequencies known as photonic band gaps [21]. Due to the small period of the modulation of the refractive index $\approx 500 \mathrm{~nm}$, photonic crystals are known as nanodevices, which allow to guide light by varying the waveguide configuration. These waveguides are usually constructed by introducing defects in a periodic structure.

Below we demonstrate that transmission of electromagnetic waves through photonic crystal waveguides can be described by a simple discrete model, which is similar to Eq. (3). We consider a two-dimensional photonic crystal created by a square lattice (with the period $a$ ) of dielectric rods in air. We study in-plane light propagation in this photonic lattice described by the electric field $E(\mathbf{x}, t)=\exp (-i \omega t) E(\mathbf{x} \mid \omega)$ polarized parallel to the rods, and reduce the Maxwell's equations to the scalar eigenvalue problem

$$
\left[\nabla^{2}+\left(\frac{\omega}{c}\right)^{2} \epsilon(\mathbf{x})\right] E(\mathbf{x} \mid \omega)=0 .
$$

A waveguide is created by replacing some of the lattice rods by the defect rods with the radius $r_{d}$, or simply by removing some rods of the lattice. To describe the structure with defects, we decompose the permittivity function $\epsilon(\mathbf{x})$ into a sum of the periodic part and the defect-induced contribution, $\epsilon(\mathbf{x})=\epsilon_{p}(\mathbf{x})+\delta \epsilon(\mathbf{x})$, and rewrite Eq. (9) in the integral form [22],

$$
E(\mathbf{x} \mid \omega)=\left(\frac{\omega}{c}\right)^{2} \int d^{2} \mathbf{y} G(\mathbf{x}, \mathbf{y} \mid \omega) \delta \epsilon(\mathbf{y}) E(\mathbf{y} \mid \omega),
$$

where $G(\mathbf{x}, \mathbf{y} \mid \omega)$ is the standard Green's function. If the radius of the defect rod $r_{d}$ is sufficiently small, the electric field $E(\mathbf{x} \mid \omega)$ inside the rod is almost constant, and the integral (10) can be easily evaluated. This allows us to derive a set of discrete equations for the electric field

$$
E_{n, m}=\sum_{k, l} J_{n-k, m-l}(\omega) \delta \epsilon_{k, l} E_{k, l}
$$

where

$$
J_{n, m}(\omega)=\left(\frac{\omega}{c}\right)^{2} \int_{r_{d}} d^{2} \mathbf{y} G\left(\mathbf{x}_{n}, \mathbf{x}_{m}+\mathbf{y} \mid \omega\right)
$$

are the frequency-dependent effective coupling coefficients and

$$
\delta \epsilon_{n, m}=\epsilon_{n, m}-\epsilon_{\mathrm{rod}},
$$

are the defect-induced changes of the lattice dielectric function, where $\epsilon_{n, m}$ is the dielectric constant of the defect rod located at the site $(n, m)$.

In general, the effective coupling coefficients $\left|J_{n, m}(\omega)\right|$ decay slow in space [23]. This slow decay introduces effective long-range interaction between different sites of the waveguide. In reality, we define a finite distance $L$ of this interaction by assuming that all coupling coefficients with the numbers $|n-k|>L$ and $|m-l|>L$ vanish. As demonstrated in Ref. [23], the case $L=6$ gives already an excellent agreement with the results of the finite-difference time-domain numerical simulations. But for many cases even the nearestneighbor interaction approximation $(L=1)$, shows a good agreement with exact results. In the case of side-coupled defects to the straight waveguide [see Fig. 2(a)], the set of equations (11) reduces exactly to (8). This drastic simplification of the original problem allows us to study the system analytically and analyze many interesting effects such as resonant light scattering. By taking into account a larger number of interaction terms will just renormalize the effect.

\section{Transmission coefficient}

To calculate the transmission coefficient for the system (8), we use the transfer matrix connecting the left and right boundaries [24]

$$
\left(\begin{array}{c}
\phi_{L} \\
\phi_{L+1}
\end{array}\right)=M\left(\begin{array}{c}
\phi_{-L} \\
\phi_{-L-1}
\end{array}\right)
$$

where $2 L$ is a characteristical width of the scattering potential.

By using the scattering boundary conditions

$$
\phi_{n}= \begin{cases}I_{0} e^{i q n}+r e^{-i q n}, & n \ll-L, \\ t e^{i q n}, & n \gg L,\end{cases}
$$

the transmission coefficient $T=\left|t^{2}\right| /\left|I_{0}\right|^{2}$ can be presented in the following form: 


$$
T=\frac{4 \sin ^{2} q}{\left|M_{11} e^{-i q}+M_{12}-M_{21}-M_{22} e^{i q}\right|^{2}} .
$$

For the $\delta$-like scattering potential (8) the transfer matrix (14) takes a very simple form

$$
M_{F}=\left(\begin{array}{cc}
a & -1 \\
1 & 0
\end{array}\right)
$$

where

$$
a=2 \cos q-\frac{V_{0}^{2}}{C} \frac{\rho_{N-1}\left(\omega_{q}\right)}{\rho_{N}\left(\omega_{q}\right)}
$$

and it defines the following transmission coefficient

$$
T=\frac{\alpha_{q}^{2}}{\alpha_{q}^{2}+1}
$$

where

$$
\alpha_{q}=\frac{c_{q} \rho_{N}\left(\omega_{q}\right)}{V_{0}^{2} \rho_{N-1}\left(\omega_{q}\right)}, \quad c_{q}=2 C \sin q .
$$

From the result (19), (20), it follows that, in general, there exist $N-1$ frequencies for the perfect transmissions (when $\alpha_{q}= \pm \infty$ ) and $N$ frequencies for the perfect reflections (when $\left.\alpha_{q}=0\right)$. Due to the specific structure of our system, the perfect transmissions are surrounded by the perfect reflections. The transmission coefficient (19), (20) is written the form similar to that of the Fano formula, and it allows us to associate the resonances with the Fano resonance.

The case of local coupling considered above is quite special, and it may be hard to realize in a real physical system. Nevertheless, it allows us to demonstrate the entire physical phenomenon and associate the resonant reflection and transmissions with the excitation of particular groups of the defects. In particular, by analyzing these results we make the following statements about the nature of the Fano resonances. (i) The frequencies of perfect reflections occur at the eigenmode frequencies of the complex $N$-site Fano defect. In order to find these frequencies, we should cut the coupling between the main array and a finite subsystem of defects and calculate a discrete spectrum of oscillatory frequencies of the isolated complex defect. This result agrees with the earlier results obtained for other types of time-periodic and spatially-localized scattering potentials [20]. (ii) The frequencies of perfect transmissions can be calculated by eliminating one degree of freedom from the a cluster of defects, as indicated in Fig. 1(a) and calculating the oscillatory eigenfrequencies of the remaining chain. Because, both the perfect reflections and perfect transmissions excite some eigenstates of the complex defect they correspond to a resonant scattering.

As an example, we consider a simple case when the complex Fano defect consists of a homogeneous chain of defects, and the energy of all sites and couplings between them are constant, $E_{m}=E$ and $V_{m}=V$. In this case, the function $\rho_{l}(\omega)$ vanishes at

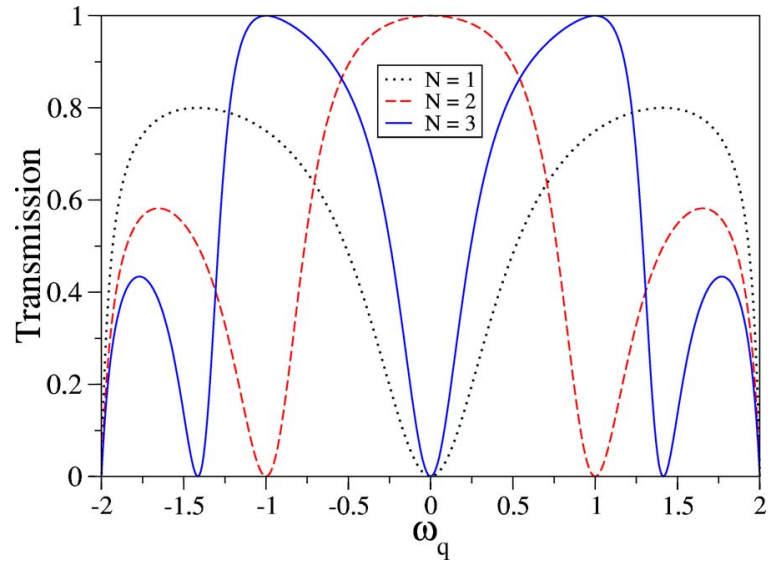

FIG. 3. (Color online) Transmission coefficient of the $N$-level Fano defect with the degenerated energies $E_{m}=E$ for $N=1,2,3$. Other parameters are $C=1, V_{m}=1$, and $E=0$.

$$
\omega_{m}=E+2 V \cos \left(\frac{m \pi}{l+1}\right), \quad m=1, \ldots, l,
$$

which leads to the corresponding resonances as shown in Fig. 3. This example shows a very interesting property of the Fano resonances. By adding or removing one additional defect we can change the transmission from zero to one for some particular frequencies. Namely, each perfect reflection of the $l$-level Fano defect with $T_{l}\left(\omega_{m}\right)=0$ becomes a perfect transmission with $T_{l+1}\left(\omega_{m}\right)=1$ after adding one more defect to the chain.

\section{Additional defects in the main array}

Now we study another important case that allows an effective engineering of the Fano resonance transmission. In particular, we study the effect of an additional defect placed in the main array. In this case, the effective equation becomes

$$
\omega \phi_{n}=C\left(\phi_{n-1}+\phi_{n+1}\right)+E_{0} \phi_{l 1} \delta_{n, l_{1}}+\frac{\rho_{N-1}(\omega)}{\rho_{N}(\omega)} V_{0}^{2} \phi_{0} \delta_{n, 0},
$$

where we assume that the $\delta$-like defect of the strength $E_{0}$ is located at the site $n=l_{1}$ and the complex $N$-level Fano defect remains at the site $n=0$ [see Fig. 1(b)].

Without losing the generality, we assume that $l_{1} \geqslant 0$, and present the transfer matrix $M$ in the following form

$$
\begin{gathered}
M=M_{\delta} M_{0}^{m} M_{F}, \\
M_{0}=\left(\begin{array}{cc}
b & -1 \\
1 & 0
\end{array}\right), \quad M_{\delta}=\left(\begin{array}{cc}
c & -1 \\
1 & 0
\end{array}\right), \\
b=2 \cos q, \quad c=b-E_{0} / C,
\end{gathered}
$$

and $m=l_{1}-1$. By using the eigenvalue expansion, we can show that 


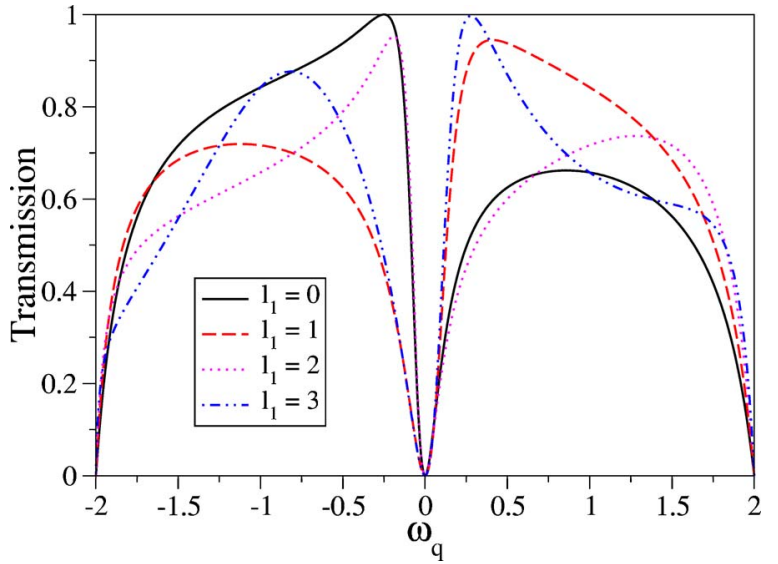

FIG. 4. (Color online) Transmission coefficient of combined Fano and $\delta$-like defects for different distances between them. Other parameters are $C=1, V_{0}=0.5, E_{0}=1$, and $E_{1}=0$.

$$
M_{0}^{m}=\frac{1}{\sin q}\left(\begin{array}{cc}
\sin [(m+1) q] & -\sin [m q] \\
\sin [m q] & -\sin [(m-1) q]
\end{array}\right),
$$

and calculate analytically the transfer matrix $M$ and the transmission coefficient (16). Again, we observe that there exist $N$ perfect reflections at the frequencies of the eigenstates of the $N$-level Fano defect, as discussed above. However, the condition for perfect transmissions becomes more complicated.

First, we consider a single Fano defect $(N=1)$ and study how the transmission depends on the distance $l_{1}$ between two defects. The transfer matrix (23) possesses the same singularity as the transfer matrix of the single Fano defect (17), which leads to the perfect reflection at the same resonant frequency (see Fig. 3). Figure 4 shows clearly that, in addition to the perfect reflection, there exists a resonant transmission due to the presence of the defect in the main array. The transmission function becomes asymmetric, and it can be described by the generalized Fano formula. We notice here a quite interesting behavior: the transmission coefficient alternates "the sign of asymmetry," i.e., $\omega_{T_{\max }}<\omega_{T_{\min }}$, for even values of $l_{1}$, and $\omega_{T_{\max }}>\omega_{T_{\min }}$, for odd values of $l_{1}$. Moreover, the maximum of the transmission does not reach one in some cases (see Fig. 4). One of the possible explanations of this effect is that a plane wave accumulates an additional phase shift propagating between the two defects, which leads to the effective decoherence and, as a result, incomplete interference. But this effect does not alter the perfect reflection, and this reveals the principal difference between the resonant reflections and the resonant transmissions associated with the Fano resonance.

In order to show more clearly the difference between the resonant reflection and the resonant transmission at the Fano resonance, we consider the case when the $\delta$-defect in the main array and the $N$-level Fano defect are coupled directly, i.e., when $l_{1}=0$, which leads to a generalized point defect in the system (22). In this case, the transmission coefficient takes the form

$$
T=\frac{4 C^{2} \rho_{N}(\omega)^{2} \sin ^{2} q}{\left[\rho_{N-1}(\omega) V_{0}^{2}+E_{0} \rho_{N}(\omega)\right]^{2}+4 C^{2} \rho_{N}(\omega)^{2} \sin ^{2} q},
$$

and the condition for perfect transmissions is

$$
\rho_{N-1}(\omega) V_{0}^{2}+E_{0} \rho_{N}(\omega)=0 .
$$

Equation (26) is a polynomial of the $\operatorname{order} N$. This means that, in general, there exists a possibility for $N$ frequencies of the perfect transmissions. Such frequencies do not coincide with the oscillatory eigenfrequencies of the $(N-1)$-defect system, as discussed above (see Fig. 1), or $(N+1)$-defect system (i.e., $N$-level Fano defect plus a single $\delta$-like defect), as one can expect. Therefore, this means that perfect transmissions do not necessarily correspond to a resonant behavior. In this case, the dependence of the transmission coefficient is flat and, therefore, the scattering potential is almost transparent. The situation changes dramatically when the frequency of perfect transmission is located very close to that of perfect reflection, i.e., $\omega_{T=1} \approx \omega_{T=0}$. In the latter case, the perfect transmission becomes resonant because it corresponds to the excitation of one of the eigenmodes of the Fano defect, which is responsible for the resonant suppression of the transmission. This physical picture explains how the Fano defect can generate, almost for the same frequency, both resonant constructive and destructive interferences creating a sharp asymmetric profile of the transmission curves. We would like to emphasize here again that the main feature of the Fano resonance is a resonant reflection rather than transmission, and the associated perfect transmission itself could be or could not be resonant.

Based on the analysis presented above, we can characterize the resonant scattering qualitatively by monitoring the strength of the excitation of the Fano defect. For that purpose, we introduce an effective power of the $N$-level Fano defect as the following norm,

$$
\Psi=\sum_{m=1}^{N}\left|\varphi_{m}\right|^{2}
$$

where, for simplicity, we assume $V_{m} \equiv V$. By using the recurrence relation (4), we express all amplitudes $\varphi_{m}$ in terms of $\varphi_{1}$,

$$
\left|\varphi_{m}\right|^{2}=\frac{V^{2 m-2} \rho_{N-m}^{2}(\omega)}{\rho_{N-1}^{2}(\omega)}\left|\varphi_{1}\right|^{2} .
$$

We use the relation (7) and employ the fact that the norm at site $n=0$ for a $\delta$-like scattering potentials is proportional to the transmission coefficient $\left|\phi_{0}\right|^{2}=T\left|I_{0}\right|^{2}$. This allows us to write the norm (27) in the following form:

$$
\Psi=\frac{T\left|I_{0}\right|^{2}}{\rho_{N}^{2}(\omega)} \sum_{m=1}^{N} V^{2 m} \rho_{N-m}^{2}(\omega) .
$$

As an example, we consider the transmission through the $(N+1)$-level $\delta$-defect, which consists of a single $\delta$-like defect in the main array and $N$-level Fano defect, as shown in Fig. 1(b). We assume the equidistant energy levels of the defects, $E_{m}=\Delta+m E, m=0, \ldots, 3$, and constant coupling, $V_{m}=V$. We observe that inside the transmission spectrum there exist two 


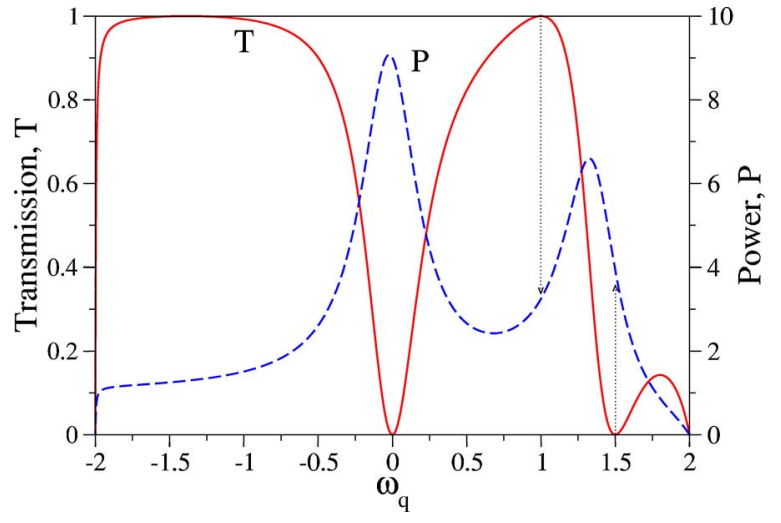

FIG. 5. (Color online) Transmission through the $N$-level Fano defect with $N=3$ and a single $\delta$-like defect in the main array for $E_{m}=\Delta+m E(m=0: N)$ and the parameters $C=1, V_{m}=1, \Delta=0.5$, and $E=0.5$. The effective norm (29) of the Fano defect is also shown to characterize the resonant excitation.

frequencies of the prefect transmission and two frequencies of the perfect reflection. One perfect transmission is resonant (at $\omega \approx 1$ ) and the other one is not (at $\omega \approx-1.5$ ). In Fig. 5 we plot also the norm of the Fano defect defined by Eq. (29). It shows that the nonresonant perfect transmission does not excite the Fano defect, and it is related to a simple constructive interference. The frequency of the other perfect transmission is located close to that of the perfect reflection, and it corresponds to a strong excitation of the Fano defect making the perfect transmission resonant. In this case, the strengths of the Fano defect excitation at the perfect transmission and perfect reflection almost coincide.

\section{NONLINEAR TRANSMISSION}

The analytical and numerical results presented above show that the resonant reflections associated with the Fano resonances are robust in the regime of a local coupling. Such reflections are observed when the frequency of the incoming wave coincides with one of the frequencies of the oscillatory eigenmodes of the attached defect chain. As a result, the defects become highly excited at the frequency of the resonant reflection. Such a specific resonant reflection can be tuned externally, and below we discuss how the presence of nonlinear defects in the discrete network may be employed to manage and tune the response of the Fano resonances.

We consider the general case of the $N$-defect Fano resonance and assume that one of the defects possesses a Kerrtype nonlinear response that can contribute as an additional nonlinear term into the system Hamiltonian,

$$
H_{N L}=H_{L}+\lambda\left|\varphi_{1}\right|^{4} \text {. }
$$

For definiteness, we choose the first defect $\varphi_{1}$ as nonlinear due to its specific role in the transmission properties and resonant reflections. Similar to the earlier studies, we expect that the presence of such a nonlinear defect will shift the positions of the perfect reflection depending on the intensity of the incoming wave [18], while the perfect transmissions will be unchanged or modified only slightly. This feature

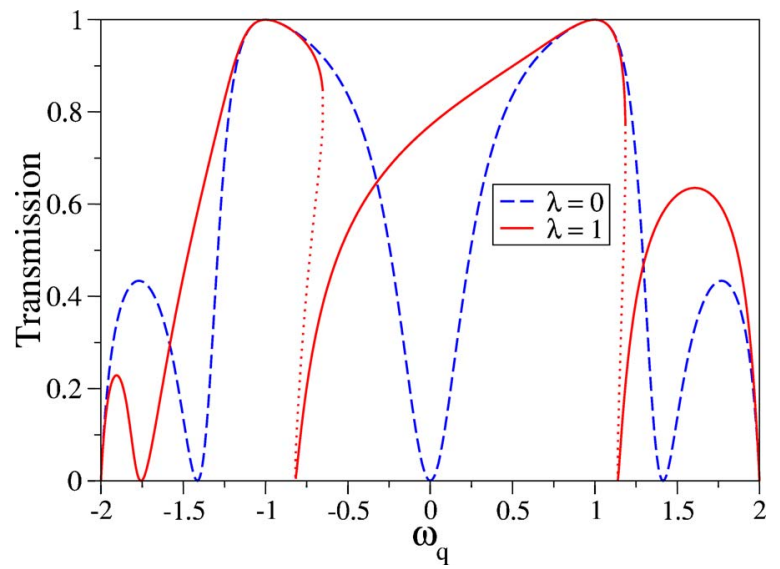

FIG. 6. (Color online) Nonlinear transmission through the $N$ $=3$ defect chain with $E_{m}=E$ coupled to the main array. Other parameters are $C=1, V_{m}=1, E=0, \lambda=1$, and $I=1$. For these parameters the shifts of the perfect reflections are large enough to show the presence of bistability. The region of the bistable transmission is indicated by the dotted line. For reference, we show the linear transmission at $\lambda=0$.

would allow us to achieve a simple tuning of the width of the asymmetric Fano resonance by changing the intensity of the incoming wave.

In the presence of the nonlinear defect, the equations of the motion can be written as follows

$$
\begin{gathered}
\omega \phi_{n}=C\left(\phi_{n-1}+\phi_{n+1}\right)+V_{0} \varphi_{1} \delta_{n, 0}, \\
\frac{\rho_{N}(\omega)}{\rho_{N-1}(\omega)} \varphi_{1}=\lambda\left|\varphi_{1}\right|^{2} \varphi_{1}+V_{0} \phi_{0},
\end{gathered}
$$

and these equations are similar to the equations of the recent paper [18] for describing the resonant transmission of a single nonlinear defect at the Fano resonance.

Using the approach developed earlier in Ref. [18], we obtain the result for the transmission coefficient

$$
T=\frac{x^{2}}{x^{2}+1},
$$

where $x$ is a real solution of the cubic equation

$$
\left(x^{2}+1\right)\left(x-\alpha_{q}\right)-\gamma_{q}=0,
$$

with the parameter $\gamma_{q}=\lambda c_{q}^{3}|I|^{2} / V_{0}^{4}$. The perfect reflection $(T$ $=0)$ takes place when there exists zero solution $x=0$ of the cubic equation (33), and this becomes possible when $\alpha_{q}$ $=-\gamma_{q}$. As a result, the presence of nonlinearity leads to a shift of the position of the perfect reflection in comparison with the case of the linear transmission at $\alpha_{q}=0$ described by Eq. (19).

When the system allows for several perfect reflections inside the transmission spectrum, the shift depends on the position and the frequencies of the resonant reflections are shifted nonuniform; see an example in Fig. 6. In contrast, the frequencies of the perfect transmission $(T=1)$ remains unchanged since they correspond to the conditions $x=\infty$ or $\alpha_{q}$ $=\infty$. As was already mentioned in Ref. [18], nonlinear trans- 
mission may become unstable and bistable when $\left|\alpha_{q}\right|^{2}>3$. Therefore, near the scattering resonances bistable transmission can be observed under some conditions, as shown in the example presented in Fig. 6.

\section{CONCLUSIONS}

We have suggested an effective way to engineer the resonant wave transmission and reflection in discrete networks through the concept of the Fano resonance management. In particular, we have analyzed the transmission properties of a linear array of interacting elements coupled to a chain of $N$ side-coupled defects and found exact analytical solutions for the transmission coefficient and the conditions for the perfect reflections and transmissions due to either destructive or constructive interferences. We have demonstrated that the nature of these reflections and transmissions can be associated with the familiar concept of Fano resonances, and we have formu- lated the basic principles of the resonant scattering management by tuning the Fano resonances. In addition, we have presented an example of a nonlinearity-tunable Fano resonance when one defect of the network possesses a nonlinear Kerr-like response. We believe our findings and the basic physical concepts of the $N$-defect transmission and reflection will be useful for many various systems where the resonant transmission can be characterized and described in terms of the Fano resonances.

\section{ACKNOWLEDGMENTS}

We thank S. Flach and S. Mingaleev for useful collaborations. This work was partially supported by the Australian Research Council through the Centre of Excellence Program. Nonlinear Physics Centre is a member of the Centre for Ultra-high bandwidth Devices for Optical Systems (CUDOS).
[1] U. Fano, Phys. Rev. 124, 1866 (1961).

[2] J. Göres, D. Goldhaber-Gordon, S. Heemeyer, M. A. Kastner, H. Shtrikman, D. Mahalu, and U. Meirav, Phys. Rev. B 62, 2188 (2000).

[3] B. R. Bulka and P. Stefanski, Phys. Rev. Lett. 86, 5128 (2001).

[4] M. E. Torio, K. Hallberg, S. Flach, A. E. Miroshnichenko, and M. Titov, Eur. Phys. J. B 37, 399 (2004); A. A. Aligia and L. A. Salguero, Phys. Rev. B 70, 075307 (2004).

[5] S. Fan and J. D. Joannopoulos, Phys. Rev. B 65, 235112 (2002).

[6] S. H. Fan, Appl. Phys. Lett. 80, 908 (2002).

[7] M. F. Yanik, S. H. Fan, and M. Soljacic, Appl. Phys. Lett. 83, 2739 (2003).

[8] A. R. Cowan and J. F. Young, Phys. Rev. E 68, 046606 (2003).

[9] S. H. Fan, W. Suh, and J. D. Joannopoulos, J. Opt. Soc. Am. B 20, 569 (2003).

[10] V. Lousse and J. P. Vigneron, Phys. Rev. B 69, 155106 (2004).

[11] S. Fan, P. R. Villeneuve, J. D. Joannopoulos, and H. A. Haus, Phys. Rev. Lett. 80, 960 (1998).

[12] S. Pereira, P. Chak, and J. E. Sipe, J. Opt. Soc. Am. B 19, 2191 (2002).

[13] A. E. Miroshnichenko and Yu. S. Kivshar, Opt. Express 13, 3969 (2005).

[14] D. N. Christodoulides, F. Lederer, and Y. Silberberg, Nature
(London) 424, 817 (2003).

[15] K. Kobayashi, H. Aikawa, S. Katsumoto, and Y. Iye, Phys. Rev. Lett. 88, 256806 (2002); K. Kobayashi, H. Aikawa, A. Sano, S. Katsumoto, and Y. Iye, Phys. Rev. B 70, 035319 (2004); T. Nakanishi, K. Terakura, and T. Ando, Phys. Rev. B 69, 115307 (2004).

[16] K. Maeda, M. Aymar, K. Ueda, H. Chiba, K. Ohmori, Y. Sato, J. B. West, K. J. Ross, and K. Ito, J. Phys. B 30, 3159 (1997); J. Tignon, M. V. Marquezini, T. Hasche, and D. S. Chemla, IEEE J. Quantum Electron. 35, 510 (1998).

[17] G. D. Mahan, Many-Particle Physics (Plenum Press, New York, 1993).

[18] A. E. Miroshnichenko, S. F. Mingaleev, S. Flach, and Yu. S. Kivshar, Phys. Rev. E 71, 036626 (2005).

[19] R. Burioni, D. Cassi, P. Sodano, A. Trombettoni, and A. Vezzani, cond-mat/0502280.

[20] S. Flach, A. E. Miroshnichenko, and M. V. Fistul, Chaos 13, 596 (2003); S. Flach, A. E. Miroshnichenko, V. Fleurov, and M. V. Fistul, Phys. Rev. Lett. 90, 084101 (2003).

[21] J. D. Joannopoulos, P. R. Villeneuve, and S. Fan, Nature (London) 386, 143 (1997).

[22] S. F. Mingaleev, Yu. S. Kivshar and R. A. Sammut, Phys. Rev. E 62, 5777 (2000).

[23] S. F. Mingaleev and Yu. S. Kivshar, Opt. Lett. 27, 231 (2002).

[24] P. Tong, B. Li, and B. Hu, Phys. Rev. B 59, 8639 (1999). 